\title{
Ab initio Calculations of the Magnetocrystalline Anisotropy in $\mathrm{UAuSb}_{2}$ Ferromagnet
}

\author{
J.A. Morkowski And A. SzAJEK \\ Institute of Molecular Physics, Polish Academy of Sciences \\ M. Smoluchowskiego 17, 60-179 Poznań, Poland
}

\begin{abstract}
The paper presents results of ab initio calculations of the magnetocrystalline anisotropy of $\mathrm{UAuSb}_{2}$, a strongly anisotropic ferromagnet below $36 \mathrm{~K}$. The total energy of the unit cell of $\mathrm{UAuSb}_{2}$ was calculated by two methods: the full potential linear muffin-tin orbitals method and by the full-potential local-orbital minimum basis band structure code. The computations were done for the following quantization directions in the tetragonal unit cell (orientations of the magnetization vector $\boldsymbol{M}$ ): [010], [001], [011], [110], [111], and [11 $\left.\frac{1}{2}\right]$. The anisotropic contribution to the total energy for various directions were fitted by the least-squares procedure to the expression for the magnetocrystalline energy suitable for tetragonal symmetry $E_{A}=K\left(\alpha_{x}^{4}+\alpha_{y}^{4}\right)+K_{2} \alpha_{z}^{2}$, where $\left(\alpha_{x}, \alpha_{y}, \alpha_{z}\right)$ is a unit vector along the direction of magnetization $\boldsymbol{M}$. The band structure calculations predict the direction [111] as the easy axis of magnetization. Values of the calculated anisotropy constants at $T=0$ are provided.
\end{abstract}

PACS numbers: 75.30.Gw; 71.20.Lp

\section{Introduction}

The class of uranium-transition metal diantimonides $\mathrm{UTSb}_{2}$, where $\mathrm{T}$ is a $3 d-$, $4 d$ - or $5 d$-transition metal, presents a variety of magnetic ordering (see [1] for comprehensive references). Many of them crystallize in a tetragonal structure of the $\mathrm{HfCuSi}_{2}$ type, of the space group $P 4 / \mathrm{nmm}$. Recently detailed study of the electronic band structure of $\mathrm{UAuSb}_{2}$ by X-ray photoemission spectroscopy and calculations was reported [2]. The compound $\mathrm{UAuSb}_{2}$ is a strongly anisotropic ferromagnet below $36 \mathrm{~K}$ [1]. Besides this qualitative statement detailed experimental data on magnetocrystalline anisotropy constants in the ferromagnetic phase are not available as yet, to our best knowledge. Ab initio electronic band structure calculations provide values of the total energy (at $T=0$ ). Calculations, as described in [2], can give total energy for various orientations of the magnetization vector $\boldsymbol{M}$. Differences between values of the total energy for various directions of $\boldsymbol{M}$ are in $\mathrm{UAuSb}_{2}$ as large as about $1 \mathrm{mRy}$ making estimates of magnetocrystalline constants (at $T=0$ ) quite feasible.

\section{Method of calculations}

In the preliminary report [3] on the magnetocrystalline anisotropy of $\mathrm{UAuSb}_{2}$ the full potential linear muffin-tin orbitals (FP LMTO) method in the form of the LmtART code (version 6.50) [4,5] was used for the band structure calculations. Details are the same as described in [2]. The exchange-correlation potential of VoskoWilk-Nusair [6] was used and the generalized gradient corrections [7] were incorporated. Obviously, the calculations were with spin polarization and spin- orbit interaction taken into account, semirelativistic approximation was used.

The tetragonal unit cell of $\mathrm{UAuSb}_{2}$ of the space group $P 4 / n m m$ accommodates two formula units. The experimental lattice constants, measured at room temperature [7], $a=0.4375 \mathrm{~nm}, c=0.9831 \mathrm{~nm}$ were employed in calculations. The atomic positions in the unit cell are $\mathrm{U}(2 \mathrm{c})=\left(\frac{1}{4}, \frac{1}{4}, 0.2438\right), \mathrm{Au}(2 \mathrm{~b})=\left(\frac{3}{4}, \frac{1}{4}, \frac{1}{2}\right)$, $\mathrm{Sb}(2 \mathrm{c})=\left(\frac{1}{4}, \frac{1}{4}, 0.6862\right), \mathrm{Sb}(2 \mathrm{a})=\left(\frac{3}{4}, \frac{1}{4}, 0\right)$. For U valence electrons comprised $5 f^{3} 6 p^{6}, 6 d^{1} 7 s^{2}$ and $6 s^{2}$ were treated as semicore ones, for $\mathrm{Au}$ the valence electrons were $5 d^{10} 6 s^{1}$ whereas for $\mathrm{Sb}$ five valence electrons $5 s^{2} 5 p^{3}$ and 10 semicore ones $4 d^{10}$ were assumed.

The values of the total energy of the unit cell for defined directions of the magnetic moment $\boldsymbol{M} / M=$ $\left(\alpha_{x}, \alpha_{y}, \alpha_{z}\right)$ had been calculated by the standard iteration procedure until achieving energy self-consistency $10^{-6}-10^{-5} \mathrm{mRy}$ and $10^{-4}-10^{-3} \mu_{\mathrm{B}}$ for magnetization. The energy self-consistency figure does not, however, guarantee the same total energy accuracy. In determining anisotropic contributions to the total energy small differences of the large total energies appear. It is reasonable to assume that the systematic errors in calculating the total energy inherent in the LMTO procedure are not particularly sensitive to variations of magnetization directions. Therefore the systematic errors are expected to cancel each other in subtracting total energies to get the anisotropic contributions.

Since the calculated by the LmtART code values of the total energy for the six directions of the quantization axis 
appeared to be fitted to expressions for the magnetocrystalline anisotropy energy only with a non-satisfying, large dispersion the whole set of the band structure calculations was repeated by a more efficient full-potential localorbital (FPLO) method [8]. For these calculations we assumed the following configurations of atoms: core (up to $5 \mathrm{p}$ electrons $)+$ valence electrons $(6 p 7 s 7 p 6 d 5 f)$ for uranium atoms, core (up to $3 d$ electrons) + valence electrons ( $4 s$ and above) for Sb atoms, and finally core (up to $4 f$ electrons) + valence electrons ( $5 s$ and above) for $\mathrm{Au}$ atoms. The calculations were performed for the reciprocal space mesh containing 196 points within the irreducible wedge of the Brillouin zone using the tetrahedron method [9] for integrations. The local spin density approximation (LSDA) exchange-correlation potential was assumed in the form proposed by Perdew and Wang [10]. The self-consistent criterion was equal to $2 \times 10^{-8} \mathrm{Ry}$ for the total energy and $10^{-6}$ of electron charge.

\section{Results and Discussion}

\subsection{LmtART}

In the ordered phase $\mathrm{UAuSb}_{2}$ is ferromagnetic. The two $\mathrm{U}$ atoms in the unit cell are crystallographically and magnetically equivalent and contribute the bulk of the total magnetic moment. The contributions from the two inequivalent pairs of $\mathrm{Sb}$ are about only $3 \%$ and the ones from $\mathrm{Au}$ are negligible. This property is illustrated by the data in Table I for the magnetization along the $c$-axis.

TABLE I

Spin and orbital magnetic moments in Bohr's magnetons (calculated for $\boldsymbol{M}$ along the $c$-axis, total energy is -240394.68592639 Ry/unit cell) per atom, are listed in parenthesis. The spin magnetic moment 0.1799 is attributed to the space between non-overlapping muffin tin spheres.

\begin{tabular}{c|c|c}
\hline \hline Atom & $\begin{array}{c}\text { Spin } \\
\text { magnetization } M_{z}\end{array}$ & $\begin{array}{c}\text { Orbital } \\
\text { magnetization } O_{z}\end{array}$ \\
\hline $\mathrm{U}(2 \mathrm{c})$ & 2.0202 & -2.9909 \\
$\mathrm{Au}(2 \mathrm{~b})$ & -0.0001 & 0.0051 \\
$\mathrm{Sb}(2 \mathrm{c})$ & -0.0284 & 0.0077 \\
$\mathrm{Sb}(2 \mathrm{a})$ & -0.0357 & 0.0021 \\
\hline interstitial region & 0.1799 & 0.0
\end{tabular}

The calculations of the total energy (per unit cell, containing 2 formula units) were performed for the following directions of quantization: [111], [11 $\left.\frac{1}{2}\right],[011]$, [001], [010], [110]. The directional cosines of $\boldsymbol{M}$ provided in Table II were used for the fitting procedures to a phenomenological formula for the magnetocrystalline anisotropy energy. The differences between the total energies calculated for different orientations of magnetization are of the order of a few mRy per unit cell. For convenience of presentation and numerical processing the total energies in the column 4 of Table II are
TABLE II

$E\left(\alpha_{x}, \alpha_{y}, \alpha_{z}\right)=($ total energy per unit cell $)-\mathrm{E}_{0}$ for directional cosines $\left(\alpha_{x}, \alpha_{y}, \alpha_{z}\right)$ of the total magnetic moment $\boldsymbol{M}=\boldsymbol{M}_{\text {spin }}+\boldsymbol{M}_{\text {orbital }}$. In the last column the energies resulting from the fit to the formula (1) with the anisotropy constants $K=1.9417$ and $K_{2}=0.5963$ (in $\mathrm{mRy} /$ unit cell). Results for LmtART calculations.

\begin{tabular}{l|l|l|c|c}
\hline \hline \multicolumn{1}{c|}{$\alpha_{x}$} & \multicolumn{1}{|c|}{$\alpha_{y}$} & \multicolumn{1}{c|}{$\alpha_{z}$} & $\begin{array}{c}E\left(\alpha_{x}, \alpha_{y}, \alpha_{z}\right) \\
{[\mathrm{mRy} / \text { unit cell }]}\end{array}$ & $\begin{array}{c}E_{f}\left(\alpha_{x}, \alpha_{y}, \alpha_{z}\right) \\
{[\mathrm{mRy} / \text { unit cell }]}\end{array}$ \\
\hline 0.70711 & 0.70711 & 0.0 & -0.04833 & -1.51449 \\
0.0 & 1.0 & 0.0 & -0.73705 & -0.54362 \\
0.0 & 0.0 & 1.0 & -0.92639 & -1.88906 \\
0.00 & 0.78847 & 0.61506 & -1.96679 & -1.50566 \\
0.62301 & 0.52590 & 0.57902 & -2.73321 & -1.84438 \\
0.57119 & 0.51878 & 0.63609 & -2.78221 & -1.89676
\end{tabular}

listed as the differences $E\left(\alpha_{x}, \alpha_{y}, \alpha_{z}\right)$ between the computed total energies (per unit cell) and the arbitrary chosen reference energy $E_{0}=-240394.685 \mathrm{Ry} /$ (unit cell), i.e. the actually computed total energy for the total (i.e. $\boldsymbol{M}_{\text {spin }}+\boldsymbol{M}_{\text {orbital }}+$ interstitial contributions) magnetic moment $\boldsymbol{M}=M\left(\alpha_{x}, \alpha_{y}, \alpha_{z}\right)$ is $E_{0}+E\left(\alpha_{x}, \alpha_{y}, \alpha_{z}\right)$. The energies $E\left(\alpha_{x}, \alpha_{y}, \alpha_{z}\right)$ in Table II were fitted to the following expression for the magnetocrystalline energy having the tetragonal symmetry, with a constant $W$ (note that $E_{0}+W$ is the isotropic part of the total energy):

$$
E\left(\alpha_{x}, \alpha_{y}, \alpha_{z}\right)=W+K\left(\alpha_{x}^{4}+\alpha_{y}^{4}\right)+K_{2} \alpha_{z}^{2} .
$$

The least-squares fitting procedure gives the following values (in mRy/unit cell): $K=1.9417, K_{2}=0.5963$, and the constant $W=-2.48536$.

The fit is by no means perfect, the average square-root deviation is 0.40 ( $\mathrm{mRy} /$ unit cell) so it is quite large. The energies calculated from Eq. (1), the column 5 of Table II, not reproduced well the true values collected in the column 4. This situation is not surprising as the results depend upon tiny differences of the large computed energy values. The anisotropy parameters $K, K_{2}$ divided by the volume of the unit cell give the anisotropy constants in the standard units $K=16 \mathrm{MJ} / \mathrm{m}^{3}, K_{2}=5 \mathrm{MJ} / \mathrm{m}^{3}$. These figures are very large. Their errors are difficult to estimate, they can be considered only as order of magnitude estimates.

A possible form of the magnetocrystalline energy in tetragonal systems could be a sum of the usual cubic term $\sim\left(\alpha_{x}^{2} \alpha_{y}^{2}+\alpha_{y}^{2} \alpha_{z}^{2}+\alpha_{z}^{2} \alpha_{x}^{2}\right)$ (or its equivalent $-\left(\alpha_{x}^{4}+\right.$ $\alpha_{y}^{4}+\alpha_{z}^{4}$ ), plus a constant) and a uniaxial term $\sim \alpha_{z}^{2}$ instead of Eq. (1). However, such a form could be fitted into the data of Table II with a slightly larger square-root deviation, 0.41. For

$E\left(\alpha_{x}, \alpha_{y}, \alpha_{z}\right)=W^{\prime}+K_{\mathrm{c}}\left(\alpha_{x}^{4}+\alpha_{y}^{4}+\alpha_{z}^{4}\right)+K_{\mathrm{u}} \alpha_{z}^{2},(2)$ the fitting procedure gives $K_{c}=0.82, K_{u}=-0.06, W^{\prime}=$ -2.03632 , all in $\mathrm{mRy} /$ unit cell. For the form (2) of the anisotropy energy the calculated values $E_{f}\left(\alpha_{x}, \alpha_{y}, \alpha_{z}\right)$ would replace the entries in the 5 th column of Table II 
by the following numbers (keeping the same order): $1.4729,-1.0623,-1.1181,-1.4688,-1.6234,-1.6248$.

However, for strongly uniaxial ferromagnet (as suggested also by the elongated shape of the crystallographic unit cell and quasi-two-dimensional shape of the Fermi surface [2]) the correct phenomenological formula for the anisotropy energy should rather be as in Eq. (1) than that of Eq. (2). In order to correctly take into account the uniaxial character of the system studied the coefficients at $\left(\alpha_{x}^{4}+\alpha_{y}^{4}\right)$ in (2) are expected to be different than the one at $\alpha_{z}^{4}$. But in analyzing magnetocrystalline anisotropy no more than two constants are generally used.

\section{2. $F P L O$}

In the second column of Table III the anisotropic parts of the total energy of a unit cell of $\mathrm{UAuSb}_{2}$ are presented in the form $E_{\mathrm{FPLO}}([h k l])=$ (total energy) $-E_{00}$, where $E_{00}=-240253.072 \mathrm{Ry}$ is an arbitrarily chosen reference energy.

TABLE III

Anisotropy energy $E_{\mathrm{FPLO}}([h k l])$ calculated by FPLO method, column 2, for the directions in column 1. The fitted values from Eq. (1) with $K=1.906$ and $K_{2}=0.925$ are collected in the column 3 .

\begin{tabular}{c|c|c}
\hline \hline $\begin{array}{c}\text { Quantization } \\
\text { direction }\end{array}$ & $\begin{array}{c}E_{\mathrm{FPLO}} \\
{[\mathrm{mRy} / \text { unit cell }]}\end{array}$ & $\begin{array}{c}E_{\mathrm{fit}} \\
{[\mathrm{mRy} / \text { unit cell }]}\end{array}$ \\
\hline$[100]$ & -0.17805 & -0.09283 \\
{$[011]$} & -1.51893 & -1.17444 \\
{$[001]$} & -0.60378 & -1.07354 \\
{$\left[11 \frac{1}{2}\right]$} & -1.24664 & -1.28269 \\
{$[111]$} & -1.53327 & -1.25939 \\
{$[110]$} & -0.84814 & -1.04501
\end{tabular}

When fitted to the formula (1) the ab initio calculated anisotropy energies provide the values of the tetragonal anisotropy constants $K=1.90617, K_{2}=0.92545$ and the constant $W=-1.99900$, all in mRy per unit cell. In the standard units the magnetocrystalline anisotropy constants are $K=16 \mathrm{MJ} / \mathrm{m}^{3}$ and $K_{2}=7.7 \mathrm{MJ} / \mathrm{m}^{3}$. The dispersion of the least square fitting is $0.12 \mathrm{mRy} / \mathrm{unit}$ cell. Thus the FPLO data are more accurate. Details are collected in Table III.

\section{Discussion}

Ab initio methods of calculating electronic band structure provide values of the total energy (per crystallographic unit cell), therefore by computing total energy of a ferromagnetic system for several directions of magnetization it is possible to estimate the magnetocrystalline anisotropy constants for $T=0$. The difficulty is that the total energy is several orders of magnitude larger than its anisotropic part. However, in the case of highly anisotropic ferromagnets, like $\mathrm{UAuSb}_{2}, a b$ initio approach seems realistic, at least for estimating orders of magnitude of the anisotropy constants. The conclusion from the both series of the band structure calculations is that the direction [111] is the easy axis of magnetization and the magnetocrystalline anisotropy is strong, the anisotropy constants $K, K_{2}$ are of the order of magnitude $16,5-6 \mathrm{MJ} / \mathrm{m}^{3}$. Magnetic measurements on $\mathrm{UAuSb}_{2}$ [11] indicate that this conclusion can be valid.

\section{Acknowledgments}

This work was supported by funds for science in years 2007-2010 as a research project no. N N202 134933.

\section{References}

[1] D. Kaczorowski, R. Kruk, J.P. Sanchez, B. Malaman, F. Wastin, Phys. Rev. B 58, 9227 (1998).

[2] J.A. Morkowski, A. Szajek, E. Talik, R. Troć, J. Alloys Comp. 443, 20 (2007).

[3] J.A. Morkowski, unpublished

[4] S.Y. Savrasov, D.Y. Savrasov, Phys. Rev. B 46, 12181 (1996).

[5] S.Y. Savrasov, Phys. Rev. B 54, 16470 (1996).

[6] S.H. Vosko, L. Wilk, M. Nusair, Can. J. Phys 58, 1200 (1980).

[7] J.P. Perdew, K. Burke, M. Ernzershof, Phys. Rev. Lett. 77, 38651 (1996).

[8] FPLO-7.00-28 (improved version of the original FPLO code by K. Koepernik, H. Eschrig, Phys. Rev. $B$ 59, 1743 (1999); http://www.fplo.de).

[9] P. Blöchl, O. Jepsen, O.K. Andersen, Phys. Rev. B 49, 16223 (1994).

[10] J.P. Perdew, Y. Wang, Phys. Rev. B 45, 13244 (1992).

[11] R. Troć, Z. Bukowski, J. Stẹpień-Damm, C. Sułkowski, Acta Phys. Pol. A 115, 186 (2009). 